\title{
Ein bronzenes Griffzungenmesser aus dem Burgenland
}

\author{
Roland Haubner', Susanne Strobl', Manuela Thurner ${ }^{2}$ und Hannes Herdits² \\ ${ }^{1}$ Institut für Chemische Technologien und Analytik, Technische Universität Wien, Wien, Österreich \\ ${ }^{2}$ Burgenländisches Landesmuseum, Eisenstadt, Österreich
}

Eingegangen 24. März 2020; angenommen 30. März 2020; online publiziert 16. April 2020

Zusammenfassung: Im Zuge der Untersuchung einer Bergbauhalde wurde in Redlschlag (Bezirk Oberwart) ein spätbronzezeitliches Griffzungenmesser gefunden.

Die werkstoffkundliche Untersuchung ergab, dass das Messer aus einer Zinnbronze mit einem Sn Gehalt von etwa $10,5 \mathrm{Gew}$. $\%$ besteht. Das metallische Gefüge zeigt eine gleichmäßige dendritische Erstarrungsstruktur, wobei die Phasen $\mathrm{Cu}_{2} \mathrm{~S}$ und $\mathrm{Cu}_{41} \mathrm{Sn}_{11}$ in den interdendritischen Bereichen vorliegen.

Da keine auffälligen Verformungen festgestellt wurden, kann angenommen werden, dass keine mechanische Nachbearbeitung des Messers erfolgte. Die Oberfläche ist mit einer gleichmäßigen Patina überzogen, in der Sn angereichert wurde.

Schlüsselwörter: Griffzungenmesser, Bronze, Metallographie

\section{A Late Bronze Age Knife from Burgenland}

Abstract: A Late Bronze Age knife was found in Redlschlag, Austria, when a Bronze Age mine dump deposit was investigated.

The materials examination showed that the knife consists of a tin bronze with a Sn content of about $10.5 \%$ by weight. The metallic structure shows a uniform dendritic solidification structure, with the phases $\mathrm{Cu}_{2} \mathrm{~S}$ and $\mathrm{Cu}_{41} \mathrm{Sn}_{11}$ in the interdendritic areas.

Since no conspicuous deformations were found, it can be assumed that the knife was not mechanically reworked. The surface is covered with an uniform patina, in which Sn has been enriched.

Keywords: Knife, Bronze, Metallography

$\overline{\text { Prof. Dr. R. Haubner ( } \bowtie)}$

Institut für Chemische Technologien und Analytik,

Technische Universität Wien,

Getreidemarkt 9/164-03,

1060 Wien, Österreich

roland.haubner@tuwien.ac.at

\section{Auffindung und archäologisches Umfeld}

Das Messer wurde bei der Untersuchung einer Bergbauhalde aufgefunden. Es gehört dem Typ Baierdorf/Velatice I an und datiert damit grob in Bronzezeit D/Ha A1. Eine eindeutige Parallele zu der vorliegenden Form mit sehr markantem Höcker am Rücken und Zierrillen knapp oberhalb der

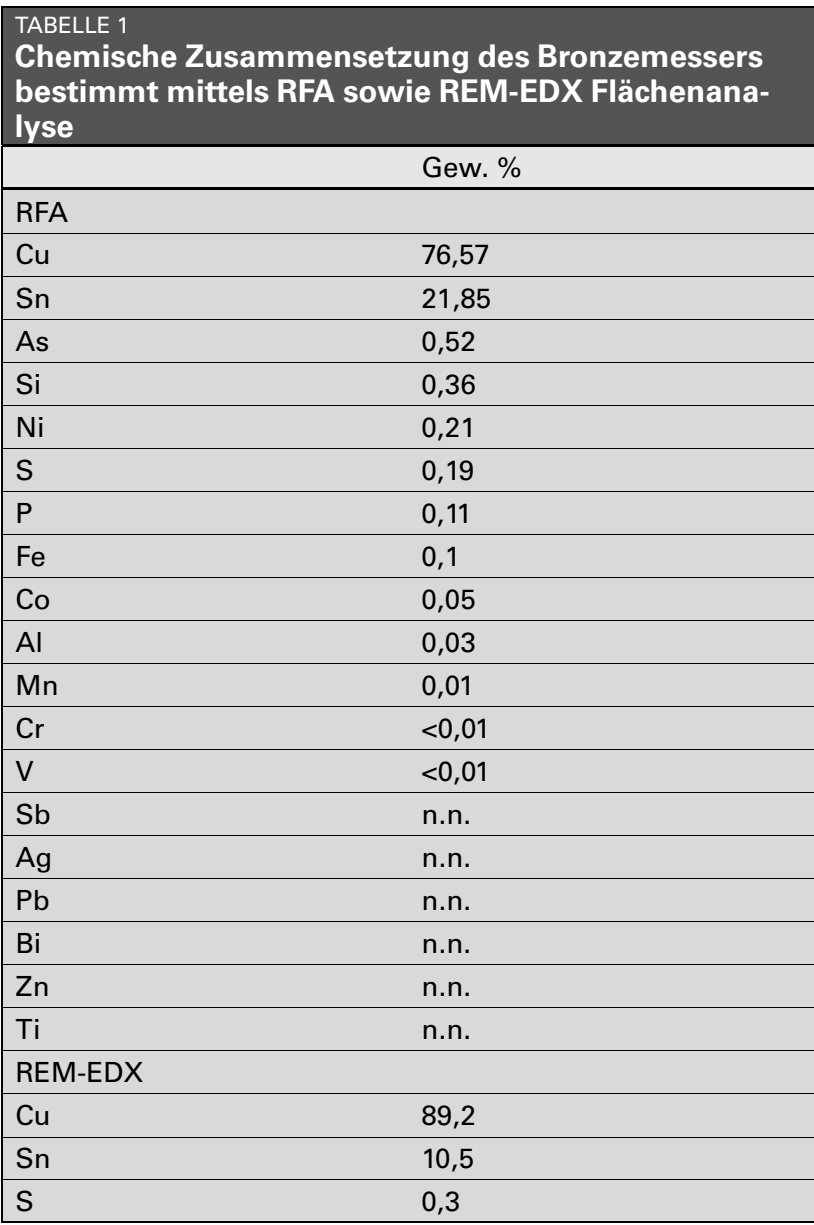


Abb. 1: Griffzungenmesser: a Originalzustand, b Position der Probenentnahme, c Schnittfläche des entnommenen Keils, d Oberfläche des Keils im 3D-DM

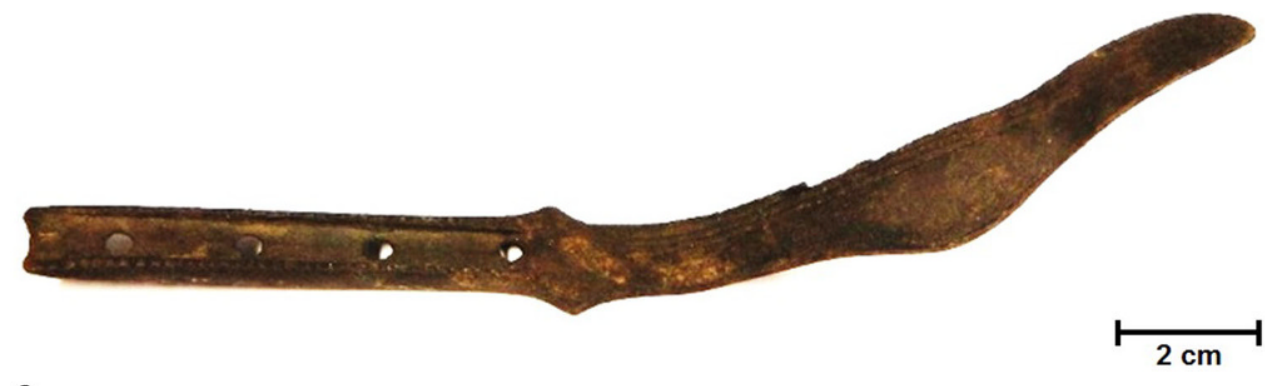

a

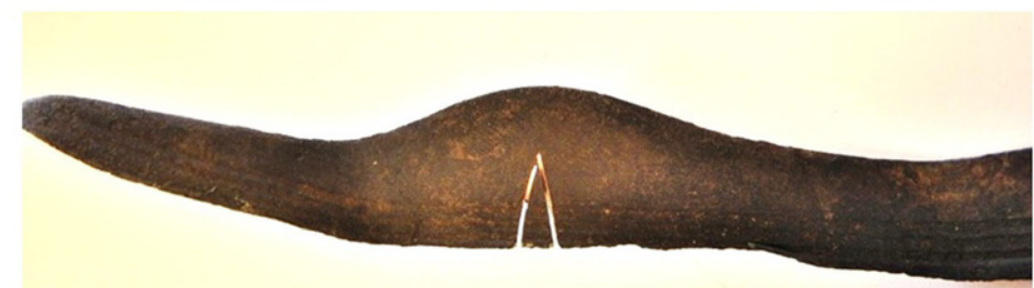

b

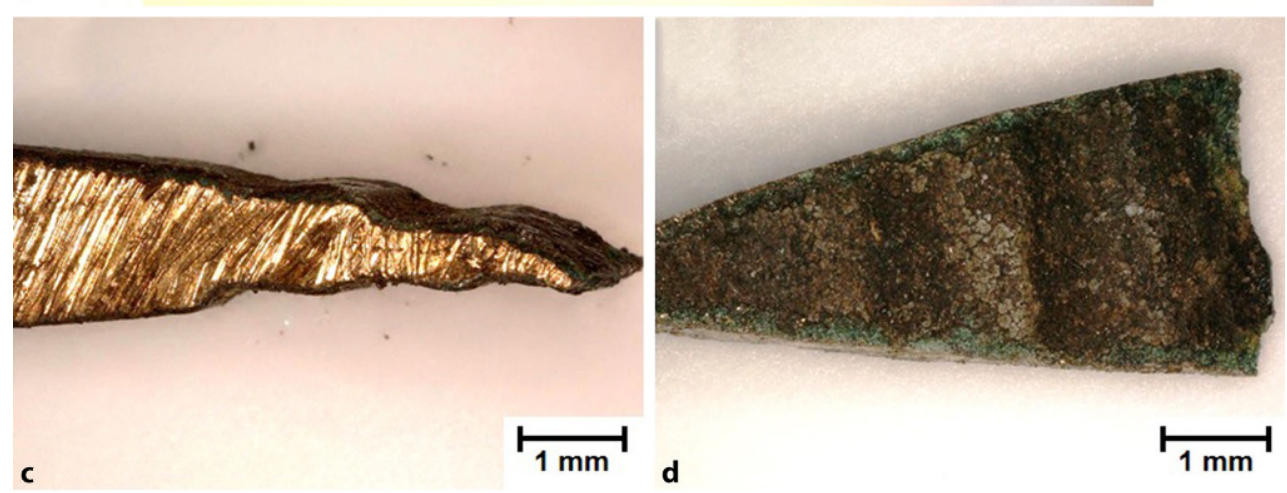

Schneide ist im mitteleuropäischen Fundgut nicht vorhanden.

Ein gutes Vergleichsstück ist aus Molln in Oberösterreich als Flussfund bekannt, jedoch liegen davon keine Werkstoffuntersuchungen vor [1].

\section{Das Griffzungenmesser}

Das Messer ist verbogen und hat eine Länge von etwa $19 \mathrm{~cm}$ (Abb. 1a).

Die Länge der Klinge beträgt ca. $10 \mathrm{~cm}$, die Breite variiert zwischen 1 und 1,6 cm. Der Klingenrücken weist einen markanten Höcker auf und die Schneide ist leicht geschweift. Entlang der Schneide verlaufen auch drei Rillen.

In der Mitte der Schneide ist diese etwas abgenutzt. Die Spitze des Messers ist abgerundet. Zwischen Klinge und Griffzunge besteht eine markante Verbreiterung $(1,9 \mathrm{~cm})$. Die Griffzunge hat eine Länge von ca. $7 \mathrm{~cm}$ und eine gleichbleibende Breite von $1,2 \mathrm{~cm}$.

Ausgeprägte Randleisten an der Griffzunge (Breite $0,5 \mathrm{~cm}$ ) sind auf ihrer gesamten Länge mit Zierkerben versehen, dürften also nicht durch organisches Material verdeckt gewesen sein. In der Griffzunge zwischen den Randleisten liegen vier Nietlöcher mit Durchmessern zwischen 0,3 und $0,4 \mathrm{~cm}$.
Aus dem breitesten Bereich der Klinge wurde ein Keil für die metallographische Untersuchung entnommen (Abb. 1b). An der Schnittfläche des Keils sind deutlich die erwähnten Rillen zu erkennen (Abb. 1c). Die Oberfläche des Messers ist überwiegend bräunlich patiniert (Abb. 1d).

\section{Metallkundliche Untersuchungen}

Der aus dem Bronzemesser entnommene Keil wurde metallographisch untersucht. Es wurden die Methoden 3D-Digitalmikroskop (3D-DM), Röntgenfluoreszenzanalyse (RFA), Metallographie, Lichtmikroskop (LOM) sowie Rasterelektronenmikroskop (REM) mit energiedispersiver Röntgenanalyse (EDX) eingesetzt. Als Ätzmittel wurden $\left(\mathrm{NH}_{4}\right)_{2} \mathrm{CuCl}_{4}$ - und $\mathrm{Klemm}$ 2-Lösungen verwendet.

Eine RFA Analyse des metallographisch präparierten Querschnitts ergab $21 \mathrm{Gew} . \% \mathrm{Sn}, 0,5 \mathrm{Gew}$. \% As, 0,2 Gew. $\%$ S, 0,2 Gew. \% Ni sowie 0,1 Gew. \% P (Tab. 1). Die Konzentrationen weiterer Spurenelemente waren unter 0,1 Gew.\%. Da 21 Gew.\% Sn in einer bronzezeitlichen Sn-Bronze unrealistisch hoch erschien und auch die metallographischen Gefüge nicht einer derartigen Legierung entsprachen, wurden Detailanalysen mittels REM-EDX durchgeführt. Diese Analysen ergaben einen Mittelwert von 10,5 Gew.\% Sn und $0,3 \mathrm{Gew}$. \% S, was auch dem vorliegenden Gefüge 
Abb. 2: Metallographisch präparierte Fläche des entnommenen Keils: a Gesamtansicht, b Schneide des Messers, c Bereich mit Korrosion im Metall, d Patina aus Malachit im LOM e Patina im polarisierten Licht Übergangsbereich Metall zu Patina im LOM, g im REM
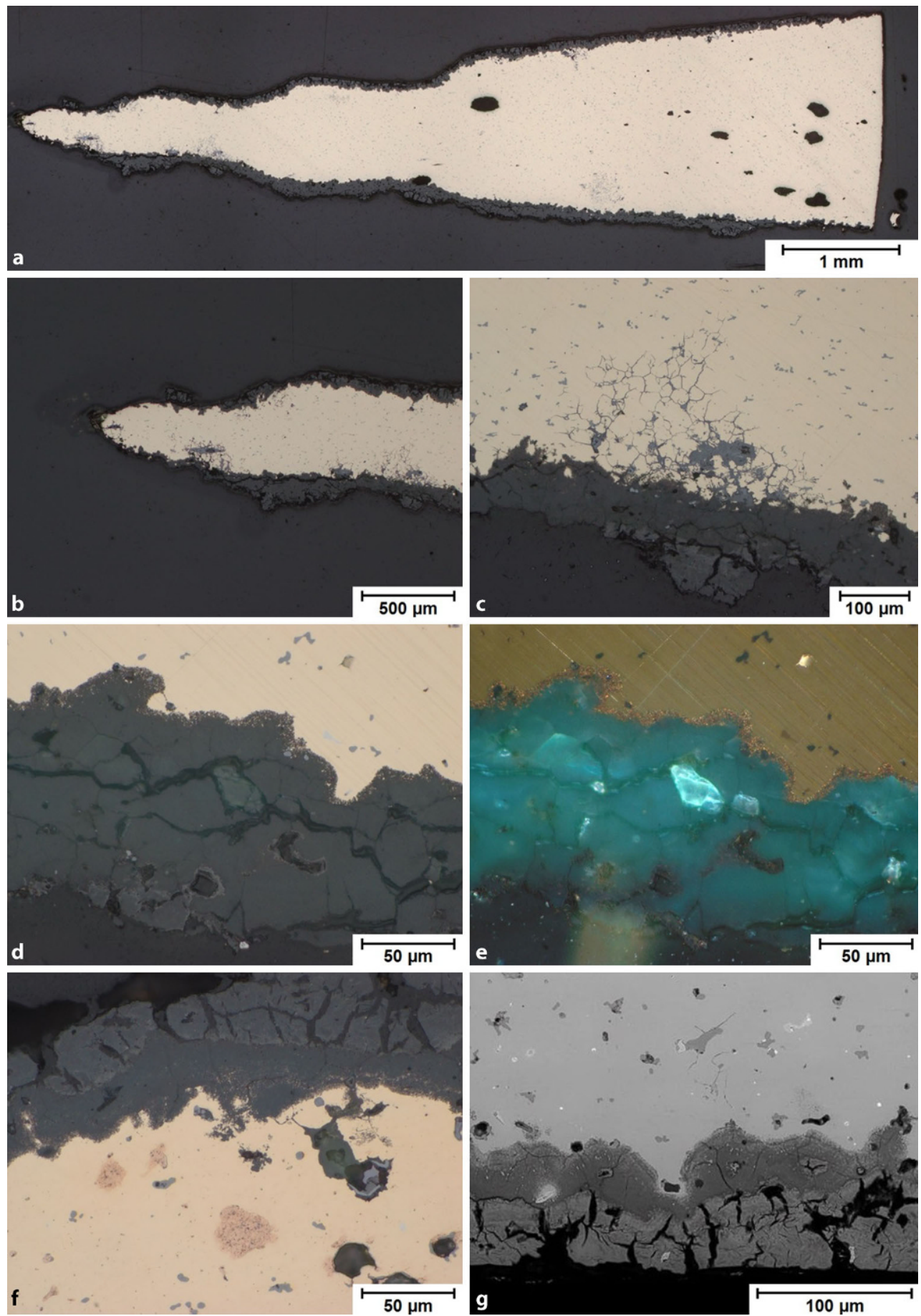
Abb. 3: Gefüge der Bronzelegierung: a Übersicht im REM, b LOM, c Cu-Sn Phase (weiß) und $\mathrm{Cu}_{2} \mathrm{~S}$ (schwarz) (REM), d Bereich mit interkristalliner Korrosion, Lunker, e im LOM undfREM
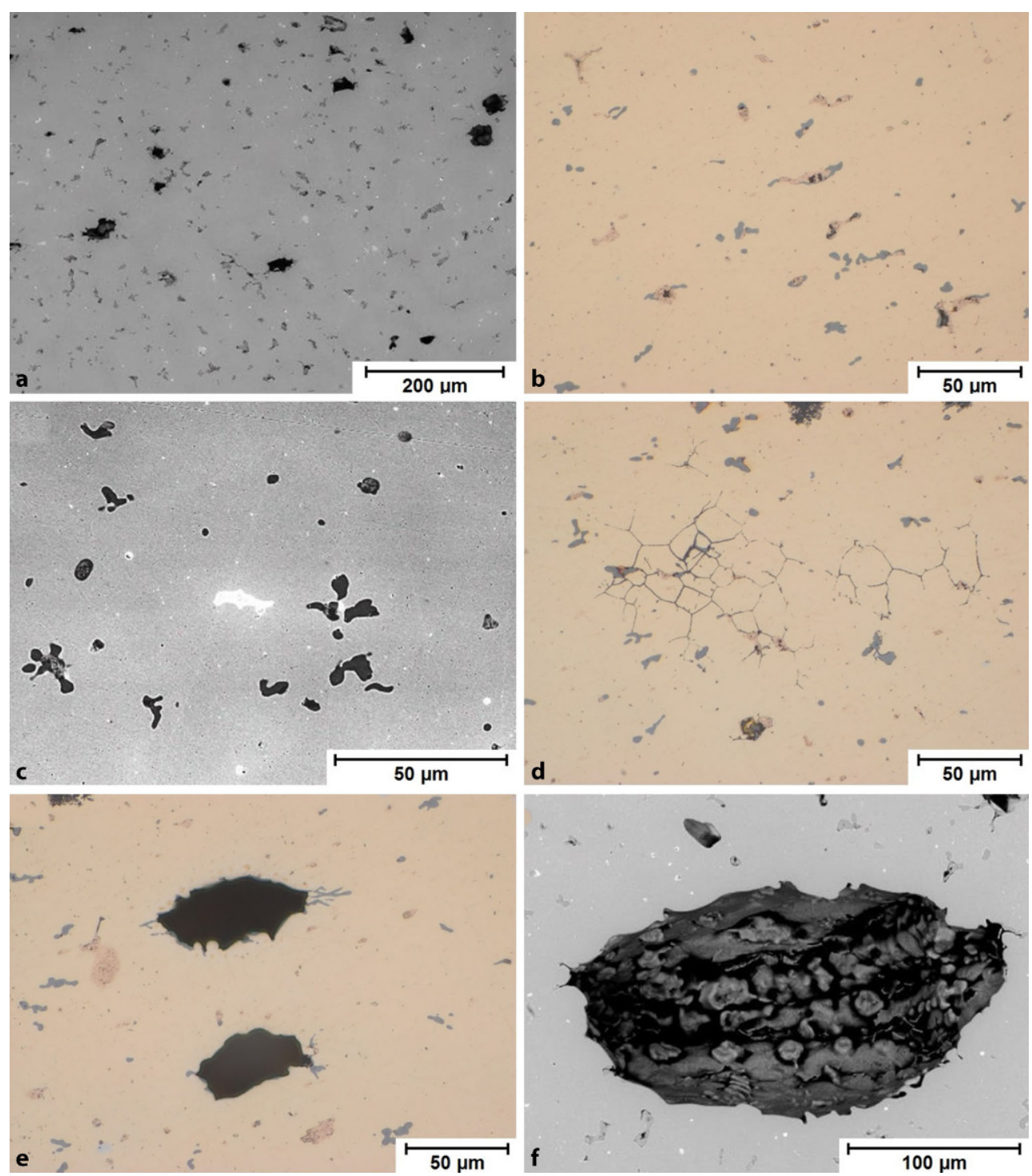

entsprach. Die Diskrepanz zwischen RFA und REM-EDX ist auf die vorliegende Patina zurückzuführen, in welcher sich Sn während der Korrosion angereichert hat [2]. Mittels RFA Messungen konnten aber die Spurenelemente erfasst werden, wobei die Anwesenheit von As und $S$ auf die Erzbasis Chalkopyrit und/oder Fahlerze hinweist [3-5].

Der metallographische Schliff des entnommenen Keils zeigt ein gleichmäßiges metallisches Gefüge mit einigen Löchern, vermutlich Lunker, die bei der Erstarrung entstanden sind (Abb. 2a). Die Bronze ist mit einer etwa 100 bis $200 \mu \mathrm{m}$ dicken Patinaschicht überzogen. In Abb. 2b ist die Schneide des Messers bei höhere Vergrößerung zu sehen, wodurch größere Einschlüsse sowie Korrosion an den Korngrenzen deutlich erkennbar sind (Abb. 2c). Die Abbildungen Abb. 2d, e zeigen Patina, welche aufgrund der grünen Farbe im polarisierten Licht als Malachit $\left(\mathrm{Cu}_{2}\left(\mathrm{CO}_{3}\right)(\mathrm{OH})_{2}\right)$ bezeichnet werden kann. $\mathrm{Cu}_{2} \mathrm{O}$, das im polarisierten Licht dunkelrot bis schwarz erschient, ist hier nicht vorhanden [2]. In einem anderen Bereich der Patinaschicht ist ein zweilagiger Aufbau zu erkennen (Abb. 2f).
Die äußere Lage erscheint kompakter, enthält aber feine Risse und die innere Lage ist feinkörniger ohne sichtbare Risse. Im REM ist der lagige Aufbau der Patina ebenfalls zu erkennen (Abb. 2g).

Im metallischen Kern des Messers sind zahlreiche Lunker und Ausscheidungen zu erkennen (Abb. 3a, b). Mittels REM-EDX Analysen an verschiedenen Ausscheidungen wurden neben $\mathrm{Cu}$ auch $\mathrm{Sn}, \mathrm{S}, \mathrm{Pb}, \mathrm{O}$ und $\mathrm{C}$ nachgewiesen. Einzelne Phasen können nicht nachgewiesen werden, aber aufgrund der Sn Konzentration von 10,5 Gew.\% ist ein eutektoides Gefüge zu erwarten. Ein kleiner Bereich eutektoiden $\mathrm{Cu}-\mathrm{Sn}$ Gefüges ist in Abb. 3c als weißer Fleck deutlich zu sehen [6]. Im Eutektoid liegen die Phasen $\mathrm{Cu}_{41} \mathrm{Sn}_{11}$ und Cu-Sn Mischkristall vor [7, 8]. Die kleinen weißen Punkte werden $\mathrm{Pb}$ zugeordnet und die schwarzen Flecken $\mathrm{Cu}_{2} \mathrm{~S}$. In Abb. 3d sind Risse an Korngrenzen des Metalls deutlich zu sehen. Ein Muster, dass die Risse bevorzugt durch bestimmte Phasen verlaufen, ist nicht zu beobachten. Die Abb. 3e, f zeigen Lunker. Im LOM ist an den Rändern der Lunker eine graue Phase zu erkennen, bei der es sich ver- 
Abb. 4: Metallographischer Schliff nach Ätzung mit Klemm 2 (LOM): a Übersicht, b, c Detailaufnahmen, die deutlich die dendritische Erstarrungsstrukturzeigen, e die $\mathrm{Cu}_{41} \mathrm{Sn}_{11}$ Phase erscheint weiß und $\mathrm{Cu}_{2} \mathrm{~S}$ braun
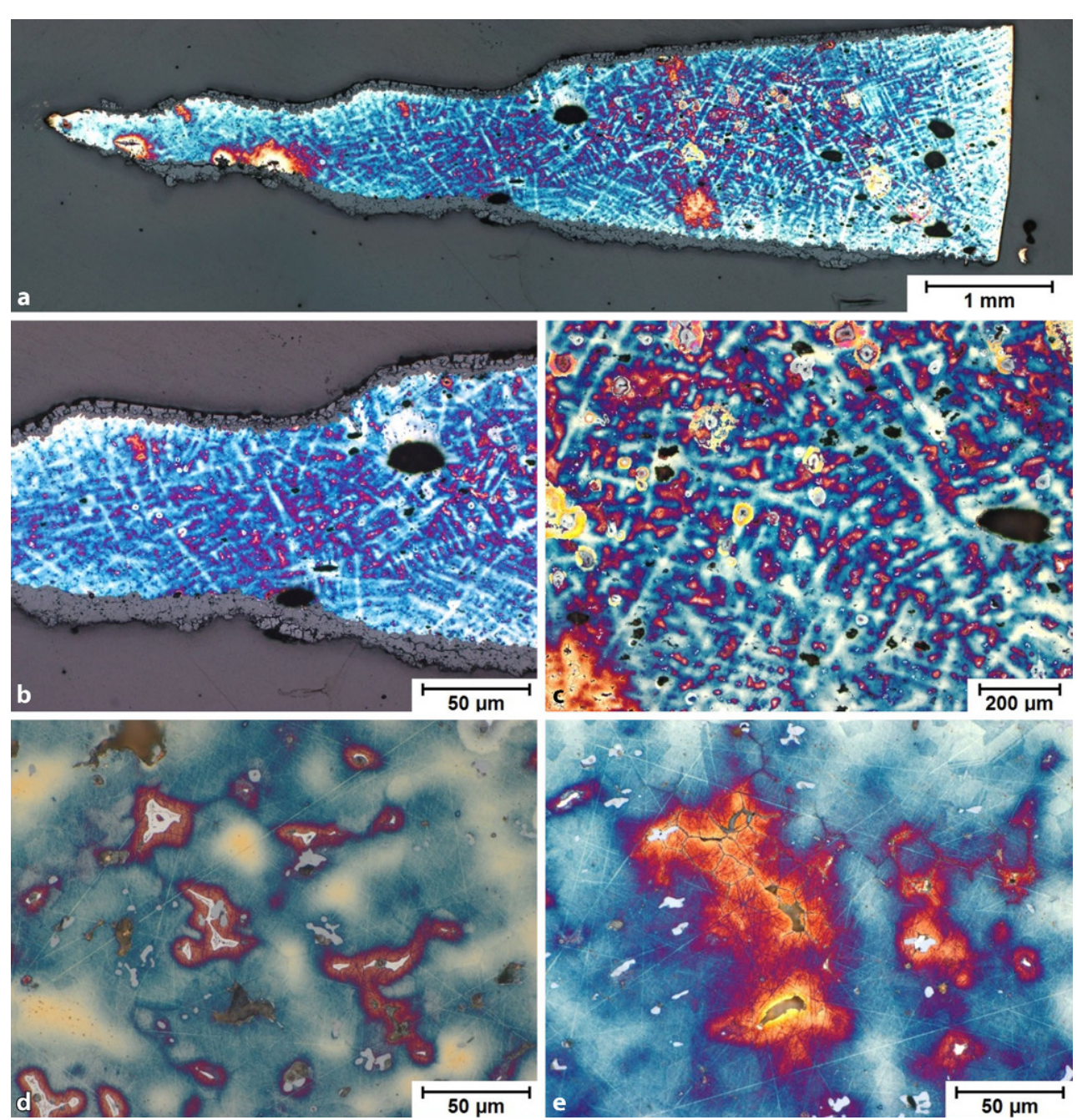

mutlich um $\mathrm{Cu}_{2} \mathrm{~S}$ handelt (Abb. 3e). Im REM ist im Lunker deutlich die dendritische Erstarrung zu erkennen (Abb. 3f).

Durch eine Ätzung mit Klemm 2-Lösung wird das metallische Gefüge blau beziehungsweise dunkelrot angeätzt (Abb. 4a). Die Überblicksaufnahme zeigt über den gesamten Probenbereich eine dendritische Erstarrungsstruktur, welche während der Erstarrung entstanden ist. Deutlich ist auch die gleichmäßige Patina an der Oberfläche zu erkennen (Abb. 4b). Die unterschiedlichen Farben der Ätzung lassen sich folgendermaßen erklären: Zuerst scheidet sich eher reineres Kupfer in Form von Dendriten aus, welche im Gefüge hellblau gefärbt sind (Abb. 4c). Mit fortschreitender Erstarrung reichern sich Verunreinigungen und Legierungselemente in der Schmelze an und werden während der Erstarrung vermehrt im Kupfer eingebaut, wodurch nach dem Ätzen eine dunkelbaue Farbe vorliegt. Die interdendritischen Bereiche, welche zuletzt erstarren und die meisten Verunreinigungen enthalten, erscheinen rot, und dort ist auch die Phase $\mathrm{Cu}_{2} \mathrm{~S}$ sowie das eutektoide $\mathrm{Cu}-\mathrm{Sn}$ Gefüge zu sehen (Abb. 4d). Die weißen Bereiche bestehen aus $\mathrm{Cu}_{41} \mathrm{Sn}_{11}$, welches von einem dunkelroten Saum umgeben sind (Abb. 4d). Ähnlich verhält es sich mit $\mathrm{Cu}_{2} \mathrm{~S}$, das aber braun gefärbt ist (Abb. 4e).
Vereinzelt sind Korngrenzen zu beobachten, und die Korngröße wird auf 10-20 $\mu \mathrm{m}$ geschätzt.

Da die Gussstruktur des Griffzungenmessers gut erhalten ist und keine ausgeprägten Verformungsstrukturen vorliegen, kann geschlossen werden, dass keine Nachbearbeitung durch z. B. Hämmern erfolgte.

\section{Die Metallurgie der Bronzezeit}

Der Übergang von der Kupferzeit zur Bronzezeit vollzog sich gleitend, wobei vermutlich die verfügbaren Kupfererze von maßgeblicher Bedeutung waren. Es kann angenommen werden, dass das erste Kupfer aus oxydischen Erzen hergestellt wurde. In weiterer Folge wurden bevorzugt Fahlerze eingesetzt, deren Verhüttung relativ einfach ist [9]. Ein zusätzlicher positiver Effekt war, dass etwas As im Kupfer verblieb und dadurch Arsenbronzen erhalten wurden, die eine etwas höhere Härte besitzt. In weiterer Folge wurde dann Chalkopyrit verhüttet, wobei hier das im Erz enthaltene Eisen als Schlacke abgetrennt werden musste [10].

Ob die Einführung der Zinnbronze gleichzeitig mit der Verhüttung von Chalkopyrit erfolgte, ist nicht nachgewie- 
sen, denn manche Zinnbronzen enthalten auch As, wie z. B. das hier untersuchte Griffzungenmesser.

Der Herstellungsort des vorliegenden Griffzungenmessers kann nicht so einfach lokalisiert werden, aber die Verarbeitung von Zinnbronze im etwa 50 km entfernten PrigglitzGasteil wurde bereits nachgewiesen [11, 12].

\section{Zusammenfassung}

Ein frühurnenfelderzeitliches (ca. 1200 v.Chr.), in einer Bergbauhalde aufgefundenes Griffzungenmesser wurde metallographisch untersucht. Es besteht aus einer Zinnbronze mit einem Sn Gehalt von etwa 10,5 Gew.\% Als weitere Elemente wurden As, S, Ni und P nachgewiesen. Im metallischen Gefüge ist ein gleichmäßiges dendritisches Erstarrungsgefüge zu erkennen. Da bei der Erstarrung der Legierung die Verunreinigungen in interdendritischen Bereichen angereichert werden, liegen auch dort die $\mathrm{Cu}_{2} \mathrm{~S}$ und $\mathrm{Cu}_{41} \mathrm{Sn}_{11}$ Phasen vor. Vermutlich erfolgte nach dem Guss keine weitere mechanische Bearbeitung, da keine auffälligen Verformungen im Gefüge festgestellt wurden. Eine gleichmäßige Patina aus Malachit bedeckt die metallische Bronze, wobei jedoch kein $\mathrm{Cu}_{2} \mathrm{O}$ festgestellt wurde. In der Patina ist aufgrund der Korrosionsmechanismen Sn angereichert.

Funding. Open access funding provided by TU Wien (TUW).

Open Access Dieser Artikel wird unter der Creative Commons Namensnennung 4.0 International Lizenz veröffentlicht, welche die Nutzung, Vervielfältigung, Bearbeitung, Verbreitung und Wiedergabe in jeglichem Medium und Format erlaubt, sofern Sie den/die ursprünglichen Autor(en) und die Quelle ordnungsgemäß nennen, einen Link zur Creative Commons Lizenz beifügen und angeben, ob Änderungen vorgenommen wurden.

Die in diesem Artikel enthaltenen Bilder und sonstiges Drittmaterial unterliegen ebenfalls der genannten Creative Commons Lizenz, sofern sich aus der Abbildungslegende nichts anderes ergibt. Sofern das betreffende Material nicht unter der genannten Creative Commons Lizenz steht und die betreffende Handlung nicht nach gesetzlichen Vorschriften erlaubt ist, ist für die oben aufgeführten Weiterverwendungen des Materials die Einwilligung des jeweiligen Rechteinhabers einzuholen.
Weitere Details zur Lizenz entnehmen Sie bitte der Lizenzinformation auf http://creativecommons.org/licenses/by/4.0/deed.de.

\section{Literatur}

1. Schmitsberger, O.: Ein neuer nordalpiner Flussfund der Urnenfelderzeit aus Oberösterreich, Jahrbuch des Oberösterreichischen Musealvereines, 152 (2007), S. 7-17

2. Haubner, R.; Strobl, S.: Long-time corrosion of a cast bronze droplet during 3000 years storage in soil, in: Proceedings EUROCORR 2015, Paper-Nr. 116 (2015), pp 1-6

3. Feuerbach, M.; Unger, H.: Die Schwefelkieslagerstätte Bernstein (Burgenland, Österreich), Archiv für Lagerstättenforschung in den Ostalpen, 9 (1969), S. 33

4. Ertl, F.; Strobl, S.; Haubner, R.: An ancient bronze ingot smelted from fahlore, Materials Science Forum, 891 (2017), pp 613-617

5. Haubner, R.; Ertl, F.; Strobl, S.: Examinations of a Bronze Ingot Made of Fahlore, Practical Metallography, 54 (2017), pp 107-117

6. Strobl, S.; Haubner, R.: Microstructural features of bronze-coated iron coins from the Roman Empire, Materials Science Forum, 782 (2014), pp 629-634

7. Schumann, H.: Metallographie, Leipzig: VEB Deutscher Verlag für Grundstoffindustrie, 1967

8. Massalski, T. B.: Binary Alloy Phase Diagrams, ASM International, Metals Park OH, 1990

9. Pernicka, E.; Lutz, J.; Stöllner, T.: Bronze age copper produced at Mitterberg, Austria, and its distribution, Archaeologia Austriaca, by Österreichische Akademie der Wissenschaften, Wien, 100 (2016), pp 19-55

10. Herdits, H.: Die ostalpine bronzezeitliche Kupfererzeugung im überregionalen Vergleich am Grundbeispiel eines Hüttenplatzes in Mühlbach/Sbg., Dissertation an der Universität Wien, Wien, 2017

11. Haubner, R.; Strobl, S.; Trebsche, P.: Analysis of Urnfield period bronze droplets formed during casting, Materials Science Forum, 891 (2017), pp 41-48

12. Haubner, R.; Strobl, S.; Trebsche, P.: Metallographic analyses from the late Urnfield period copper mining settlement at Prigglitz-Gasteil in Lower Austria, in: Alpine Copper II - Alpenkupfer II - Rame delle Alpi II - Cuivre des Alpes II. New Results and Perspectives on Prehistoric Copper Production, in Turck, R.; Stöllner, T.; Goldenberg, G. (Eds): Der Anschnitt, Beiheft, 42 (2019), pp 323-332

Hinweis des Verlags. Der Verlag bleibt in Hinblick auf geografische Zuordnungen und Gebietsbezeichnungen in veröffentlichten Karten und Institutsadressen neutral. 